\title{
First Principles Study of Work Functions of Double Wall Carbon Nanotubes
}

\author{
Bin Shan \\ Department of Applied Physics, Stanford University, Stanford CA, USA, 94305-4040 \\ Kyeongjae Chd* \\ Department of Mechanical Engineering, \\ Stanford University, Stanford CA, USA, 94305-4040
}

(Dated: July 4, 2018)

\begin{abstract}
Using first-principles density functional calculations, we investigated work functions (WFs) of thin double-walled nanotubes (DWNTs) with outer tube diameters ranging from $1 \mathrm{~nm}$ to $1.5 \mathrm{~nm}$. The results indicate that work function change within this diameter range can be up to $0.5 \mathrm{eV}$, even for DWNTs with same outer diameter. This is in contrast with single-walled nanotubes (SWNTs) which show negligible WF change for diameters larger than $1 \mathrm{~nm}$. We explain the WF change and related charge redistribution in DWNTs using charge equilibration model (CEM). The predicted work function variation of DWNTs indicates a potential difficulty in their nanoelectronic device applications.
\end{abstract}

PACS numbers: 31.15.Ar, 72.80.Rj, 73.30.+y

*Electronic address: kjcho@stanford.edu 
Double-walled nanotubes (DWNTs) have attracted considerable attention due to their novel electronic and mechanical properties 1]. Compared to single-walled nanotubes (SWNTs), they have higher mechanical stiffness and greater thermal stability which may be of benefit in field emission devices 2]. Their double shell structure offers the possibility of shielding the inner tube from external perturbations [3]. Such advantages make them very interesting for certain device applications.

Utilization of DWNTs in early days were largely hindered by the unwanted carbon nanomaterials generated at the same time with DWNTs during fabrication processes. Over past few years, substantial improvements have been achieved in fabrication and purification techniques of DWNTs. Synthesis of high-quality DWNTs in large scale has been realized using high temperature pulsed arc discharge technique 4] and catalytic chemical vapor deposition [5]. In a more recent experiment, production of DWNTs with experimental yields more than $95 \%$ has been reported 6$]$. Thin DWNTs has also been successfully fabricated by by filling $C_{60}$ molecules in SWNTs followed by electron beam irradiation, yielding DWNTs with diameter distribution around $13 \sim 14 \AA[7]$. In addition, it has now become possible to identify atomic correlations between adjacent graphene layers using high-resolution transmission electron microscopy [8], enabling an unambiguous classification of DWNTs. All these point toward a possible application of DWNTs in nanoelectronic devices.

Recently, the potential use of DWNTs as channel materials for field-effect transistors (FETs) has been successfully demonstrated [9], with better subthreshold swing factor as compared to SWNTs. Also, due to different inner/outer tube combinations and inter-layer interactions, DWNTs-based FET yields richer transistor characteristics [10]. For further large-scale integration of DWNT-based FETs, an understanding of DWNT work function is essential, since even work function change on the order of $0.1 \mathrm{eV}$ may lead to substantial changes in device characteristics [1]].

The encapsulation of molecules such as $C_{60}$ inside SWNTs are known to modify the electronic properties of carbon nanotubes 12]. Due to their concentric double layer structures, ultra small diameter tubes can be encapsulated as the inner shell of DWNTs 13]. Nanotubes with diameters $\sim 4 \AA$ have already been observed in confined channels or as the inner shell of multiwall nanotubes 14, 15, 16]. The inner tubes in thin DWNTs usually feature high surface curvature and are shown to have large variations in work function[17]. There have been some previous theoretical investigations on charge redistribution in selected DWNTs [13, 18]. 
However, as far as we know, there have been no systematic study on the work functions of DWNTs and it remains unclear how the encapsulation of ultra small inner tubes affect the work functions of DWNTs.

In this study, we report first-principles work function calculations for two series of DWNTs consisting of either $(\mathrm{m}, \mathrm{m}) @(\mathrm{n}, \mathrm{n})$ pairs or $(\mathrm{m}, 0) @(\mathrm{n}, 0)$ pairs $(m<n)$, with the first chiral index representing the inner tube and the latter representing the outer tube. The diameters of the DWNTs we studied range from $1 \mathrm{~nm}$ to $1.5 \mathrm{~nm}$, within which work function variation is most significant. It was found that the work function variations can be up to $0.5 \mathrm{eV}$, even for DWNTs with similar outer diameter. This can be a significant value in device physics. The origin of work function modulations in DWNTs, as well as the charge redistribution are shown to be correlated with the type of the inner shell, and can be qualitatively understood within the framework of CEM. Some issues in the characterization and measurements of thin DWNTs are also discussed.

The work function was calculated using the standard procedure by taking the difference between the vacuum level $\phi$ and the Fermi level $E_{f}$. The vacuum level $\phi$ is determined from the average potential at the center of the vacuum region where it approaches a constant. We have excluded the slowly decaying exchange-correlation part of the potential in the vacuum region to achieve better convergence [19]. $E_{f}$ was placed at the midgap in the case of a semiconducting DWNT. The calculation was done using Vienna Ab-initio Simulation Package (VASP) [20] within local density approximation (LDA). 30 k-points were used along the nanotube's one dimensional Brillouin zone which was tested to give good convergence. In all simulations, an orthorhombic unit cell with cross-sectional dimensions of $(35 \times 35) \AA^{2}$ was used. With this unit cell size, the minimum separation between periodic tube images was $>18 \AA$, which ensures the corresponding work function converges to a constant value. KohnSham single-electron wave functions were expanded by 285768 planewaves with an energy cut-off of $286.6 \mathrm{eV}$. For the LDA exchange-correlation potential, we have used a functional form fitted to the Monte Carlo results of homogeneous electron gas [21]. Conjugate-gradient method was used for both electronic structure calculation and geometry optimization. The DWNTs were assumed to be fully relaxed when the force on each atom was less than $0.05 \mathrm{eV} / \AA$.

We first discuss the stability and optimal inter-wall spacing for DWNTs. The energetics with respect to inter-wall spacing was calculated by fixing the inner tube diameter and 
varying the diameter of the outer tube. Fig. 1(a) shows the heat of formation for selected DWNTs $\left[\Delta E=E(D W N T)-E(S W N T)_{\text {outer }}-E(S W N T)_{\text {inner }}\right]$ with different inter-wall spacing. It was found that most energetically favorable outer tube for $(3,3)$ and $(4,0)$ tubes are $(8,8)$ and $(13,0)$ tubes, respectively. For the two series of DWNTs we studied within diameter range, the most stable inner-outer combination is consistently $(\mathrm{m}, \mathrm{m}) @(\mathrm{~m}+5, \mathrm{~m}+5)$ for armchair pairs and $(\mathrm{m}, 0) @(\mathrm{~m}+9,0)$ for zigzag pairs. The optimal inter-wall spacings corresponding to such combinations are close to the inter-layer spacing of graphite $(3.35 \AA)$, possibly due to structural similarities between graphene sheet and nanotubes. This value of inter-wall spacing is consistent with other first-principles calculations 22] and experimental values from scanning tunneling microscope measurements[23]. Even though in real fabrication processes, DWNTs with non-optimal inter-wall spacing are also expected to exist, those with optimal inter-wall spacing is more energetically favorable and would prevail in the final product. Moreover, similar correlation to the inner tube chirality was seen in DWNTs with non-optimal inter-wall spacing. Thus, in the following, we focus our discussion on work functions of DWNTs with optimal inter-wall spacing.

Figure 1(b) summarizes work functions of various DWNTs with optimal inter-wall spacing, plotted against the diameter of the outer tube. Also shown on the graph are work functions of SWNTs, indicated by the dotted line. As can be seen from the graph, there is essentially no diameter dependence of work functions for SWNTs larger than 1nm (Type I SWNT). However, different work functions of DWNTs can be observed in the diameter range from $1 \mathrm{~nm}$ to $1.5 \mathrm{~nm}$, for up to $0.5 \mathrm{eV}$. More specifically, $(\mathrm{m}, 0) @(\mathrm{n}, 0)$ DWNTs show a general decrease trend in work functions while those of $(m, m) @(n, n)$ pairs show little variation. This indicates that the work function difference is primarily due to the presence of the inner ultra small diameter tube (Type II SWNT), which are known to have substantial differences in work functions 17]. In particular, when the inner shell of the DWNTs are of $(\mathrm{m}, 0)$ zigzag type, the overall work function of the DWNT was increased due to the higher work function of the inner tube.

The qualitative feature of the work function variations in DWNTs can be understood by the CEM, which has been successfully applied to molecular dynamics simulations [24]. Generally speaking, when two materials systems with different chemical potentials (minus of work function) comes into contact, chemical potentials are equalized by electron transfer from higher chemical potential system to lower potential system. The total electrostatic 
energy of such a system can be written, neglecting higher order terms, as

$$
\begin{aligned}
E_{\text {tot }}\left(q_{1}, q_{2}, \ldots, q_{N}\right) & =\sum_{i}^{N}\left(E_{i}^{0}+\left(\frac{\partial E}{\partial q_{i}}\right) q_{i}+\frac{1}{2}\left(\frac{\partial^{2} E}{\partial q_{i}^{2}}\right) q_{i}^{2}\right)+\frac{1}{2} \sum_{i \neq j} V_{i j}\left(q_{i}, q_{j}\right) \\
& =\sum_{i}^{N}\left(E_{i}^{0}+\chi_{i}^{0} q_{i}+\frac{1}{2} J_{i}^{0} q_{i}^{2}\right)+\frac{1}{2} \sum_{i \neq j} V_{i j}\left(q_{i}, q_{j}\right)
\end{aligned}
$$

Where $E_{i}^{0}$ is the energy of neutral atom $i,-e q_{i}$ is the excess electrons on atom $i, \chi_{i}^{0}$ is the electronegativity (minus of work function), $J_{i}^{0}$ is the atomic hardness, and $V_{i j}$ is the Coulombic interaction between atoms $i$ and $j$. In the context of DWNTs, we can view the DWNT as a giant 'molecule', with inner and outer tube being two artificial 'atoms' $A$ and $B$. Taking the derivative of $E_{\text {tot }}$ with respect to $q_{i}$, we arrive at the following equations for chemical potential and charge transfer:

$$
\begin{gathered}
\chi_{A}(q)=\chi_{A}^{0}+J_{A}^{0} q+\frac{q}{2 C} \\
\chi_{B}(-q)=\chi_{B}^{0}-J_{B}^{0} q-\frac{q}{2 C}
\end{gathered}
$$

where $C=\frac{2 \pi \varepsilon}{\ln (b / a)}$ is the unit length capacitance between two coaxial cylinders, with $a$ and $b$ being the inner and outer tube diameter, respectively. The dielectric constant of the nanotube is set to 1 25]. Under equilibrium, the electrochemical potential of the inner tube and outer tube must equal. By equating $\chi_{A}(q)$ and $\chi_{B}(-q)$, we have the following first-order solution for the final chemical potential and charge transfer for DWNTs:

$$
\begin{gathered}
q=\frac{\chi_{B}^{0}-\chi_{A}^{0}}{J_{A}^{0}+J_{B}^{0}+1 / C} \\
\chi=\frac{J_{B}^{0}+\frac{1}{2 C}}{J_{A}^{0}+J_{B}^{0}+\frac{1}{C}} \chi_{A}^{0}+\frac{J_{A}^{0}+\frac{1}{2 C}}{J_{A}^{0}+J_{B}^{0}+\frac{1}{C}} \chi_{B}^{0}
\end{gathered}
$$

It can be seen that the direction and amount of charge transfer is directly related to the work function difference between the inner and outer tubes, which is confirmed by subsequent charge redistribution analysis. The final work function of DWNT is a linear combination of work functions of the inner and outer tubes. Due to the small inter-layer spacing and the resulting large $1 / C$ term, the work function of DWNT can be roughly approximated by the average of work function of inner and outer tubes. Table \lists work functions 
predicted by the CEM as well as those from first-principles calculations. The general trend of work function change predicted from CEM is in good agreement with the first-principles calculations, with the largest error $\sim 0.2 \mathrm{eV}$.

The charge redistribution in DWNTs are calculated by subtracting the charge distribution of isolated inner and outer tubes from the self-consistent charge distribution, $\Delta \rho=\rho(D W N T)-\rho($ inner $)-\rho($ outer $)$. Fig 2(a,c,e) and $(\mathrm{b}, \mathrm{d}, \mathrm{f})$ shows the charge accumulation regions and charge depletion regions, respectively. In $(4,0) @(13,0)$ DWNT, the work function of the inner tube is $\sim 1 \mathrm{eV}$ higher than that of the outer tube. Due to this large difference in work function, the amount of charge transfer is also more significant than in other DWNTs, as indicated by the dense contour lines in Fig. 2(a) and (b). The direction of charge transfer can also be clearly identified. The accumulated charge mainly locates on the inner $(4,0)$ tube region rather in in the empty inter-tube region. Such charge transfer pattern has also been observed in $(5,0) @(14,0)$ tubes. This indicates that provided large enough work function difference, the direction of charge transfer can be predicted from SWNT work functions, consistent with one's physical intuition. In the case of $(8,0) @(17,0)$ and $(3,3) @(9,9)$ DWNTs, the work function difference of inner and outer tube is not as large and the direction of charge transfer is more subtle due to the presence of inter-wall interaction. The depleted charge from the $\pi$ electron system accumulates in the intertube region, with characteristic similar to the interlayer state in graphite systems. Such interlayer state in DWNTs has also been observed in other first-principles studies [18]. The amount of charge transfer is also smaller as evident by the sparse contour lines on Fig. 2(c-f).

Finally, we discuss some possible issues in the characterization of thin DWNTs. Fig. 3 shows the site-projected density of states (DOS), i.e. local DOS integrated within spheres of Wigner-Seitz radius centered on each ion, for the above three DWNTs. In $(m, 0) @(n, 0)$ DWNTs such as $(4,0) @(13,0)$, the outer tube is semiconducting with low WF and the inner tube is metallic with high WF due to $\sigma-\pi$ hybridization[26]. When they form DWNT, substantial charge transfer from the outer tube to the inner tube leads to hole doping of the outer tube. Even though the band gap of the semiconducting outer tube is largely retained despite of the inter-wall interaction, the Fermi level is below its valence band edge, giving rise to a finite DOS at the fermi energy (Fig. 3(a)). For those DWNTs with ultra small zigzag SWNTs as inner shell, their work functions are thus expected to be $0.2 \sim 0.5 \mathrm{eV}$ higher than larger diameter DWNTs. As the DWNT diameter gets larger, such as for a 
$(8,0) @(17,0)$ pair, the work functions of the inner and outer tube are comparable and their individual electronic structures are less perturbed. The DWNT is a semiconductor with a reduced band gap as compared to band gaps of both the outer and inner tube (Fig. [3(b)). (3,3)@(8,8) DWNT presents an interesting case. Even though it has almost the same diameter as $(4,0) @(13,0)$ one and is also metallic, its electronic structure around the fermi level is considerably different. Due to the presence of inter-wall interaction, states at the fermi level are mostly localized in the inner tube with tails extending to the outer shell (Fig. [3(c)). Its work function is about $0.5 \mathrm{eV}$ lower than that of $(4,0) @(13,0)$ DWNT, due to the

higher WF of the inner tube[17]. These differences are likely to affect the transport and scanning tunneling microscope measurements of DWNTs.

In conclusion, we have systematically studied the work functions of two series of DWNTs. It was found that the work function differences of DWNTs in a narrow diameter distribution $(1.0 \mathrm{~nm} \sim 1.5 \mathrm{~nm})$ can be up to $0.5 \mathrm{eV}$. The general trend of the work function change and charge redistribution are successfully explained by the CEM. These findings provides useful insight into the work function change in DWNTs which may be used to develop new ways of engineering electronic structures of DWNTs.

\section{Acknowledgments}

This work is supported by NSF grant on Network for Computational Nanotechnology $(\mathrm{NCN})$. Part of the calculations are done at San Diego Supercomputing Center.

[1] C. Y. Wei, K. Cho, and D. Srivastava, Phys. Rev. B 67, 115407 (2003).

[2] Y. W. Son, S. Oh, J. Ihm, and S. Han, Nanotechnology 16, 125 (2005).

[3] M. Endo, T. Hayashi, H. Muramatsu, Y. A. Kim, H. Terrones, M. Terrones, and M. S. Dresselhaus, Nano Lett. 4, 1451 (2004).

[4] T. Sugai, H. Yoshida, T. Shimada, T. Okazaki, and H. Shinohara, Nano. Lett. 3, 769 (2003).

[5] E. Flahaut, R. Bacsa, A. Peigney, and C. Laurent, Chem. Commun. 12, 1442 (2003).

[6] M. Endo, H. Muramatsu, T. Hayashi, Y. A. Kim, M. Terrones, and M. S. Dresselhaus, Nature 433, 476 (2005).

[7] B. W. Smith, M. Monthioux, and D. E. Luzzi, Nature(London) 396, 323 (1998). 
[8] A. Hashimoto, K. Suenaga, K. Urita, T. Shimada, T. Sugai, S. Bandow, H. Shinohara, and S. Iijima, Phys. Rev. Lett. 94, 045504 (2005).

[9] T. Shimada, T. Sugai, Y. Ohno, S. Kishimoto, T. Mizutani, H. Yoshida, T. Okazaki, and H. Shinohara, Appl. Phys. Lett. 84, 2412 (2004).

[10] S. Wang, X. L. Liang, Q. Chen, Z. Y. Zhang, and L. M. Peng, J. Phys. Chem. B 109, 17361 (2005).

[11] J. Guo and M. S. Lundstrom, IEEE Trans. Electron Devices 49, 1897 (2002).

[12] Y. M. Cho, S. W. Han, G. Kim, H. Lee, and J. Ihm, Phys. Rev. Lett. 90, 106402 (2003).

[13] Y. L. Mao, X. H. Yan, Y. Xiao, J. Xiang, Y. R. Yang, and H. L. Yu, Phys. Rev. B 71, 033404 (2005).

[14] Z. M. Li, Z. K. Tang, H. J. Liu, N. Wang, C. T. Chan, R. Saito, S. Okada, G. D. Li, J. S. Chen, N. Nagasawa, and S. Tsuda, Phys. Rev. Lett. 87, 127401 (2001).

[15] L. C. Qin, X. Zhao, K. Hirahara, Y. Miyamoto, Y. Ando, and S. Iijima, Nature 408, 50 (2000).

[16] N. Wang, Z. K.Tang, G. D. Li, and J. S. Chen, Nature 408, 51 (2000).

[17] B. Shan and K. Cho, Phys. Rev. Lett. 94, 236602 (2005).

[18] Y. Miyamoto, S. Saito, and D. Tomanek, Phys. Rev. B 65, 041402 (2002).

[19] T. C. Leung, C. L. Kao, W. S. Su, Y. J. Feng, and C. T. Chan, Phys. Rev. B 68, 195408 (2003).

[20] G. Kresse and J. Furthmuller, Comp. Mat. Sci. 6, 15 (1996).

[21] D. M. Ceperley and B. J. Alder, Phys. Rev. Lett. 45, 566 (1980).

[22] J. C. Charlier and J. P. Michenaud, Phys. Rev. Lett. 70, 1858 (1993).

[23] M. H. Ge and K. Sattler, Science 260, 515 (1993).

[24] A. K. Rappe and W. A. Goddard, J. Phys. Chem. 95, 3358 (1991).

[25] F. Leonard and J. Tersoff, Appl. Phys. Lett. 81, 4835 (2002).

[26] X. Blase, L. X. Benedict, E. L. Shirley, and S. G. Louie, Phys. Rev. Lett. 72, 1878 (1994). 
TABLE I: Work Functions of selected DWNTs

\begin{tabular}{|c|c|c|c|c|c|}
\hline DWNT & $\begin{array}{c}\text { Tube diameter } \\
(\AA)\end{array}$ & $\begin{array}{l}\text { Outer Shell } \\
\text { WF }(e V)^{*}\end{array}$ & $\begin{array}{c}\text { Inner Shell WF } \\
(\mathrm{eV})^{*}\end{array}$ & $\begin{array}{c}\text { WF from CEM } \\
(\mathrm{eV})\end{array}$ & $\begin{array}{c}\text { WF from DFT } \\
(\mathrm{eV})\end{array}$ \\
\hline$(4,0) @(13,0)$ & 10.32 & 4.70 & 5.95 & 5.32 & 5.13 \\
\hline$(5,0) @(14,0)$ & 11.11 & 4.66 & 5.10 & 4.88 & 5.00 \\
\hline$(6,0) @(15,0)$ & 11.91 & 4.68 & 4.99 & 4.84 & 4.80 \\
\hline$(7,0) @(16,0)$ & 12.70 & 4.69 & 5.10 & 4.90 & 4.90 \\
\hline$(8,0) @(17,0)$ & 13.49 & 4.69 & 4.80 & 4.74 & 4.77 \\
\hline$(3,3) @(8,8)$ & 11.00 & 4.68 & 4.50 & 4.59 & 4.64 \\
\hline$(5,5) @(10,10)$ & 13.75 & 4.68 & 4.63 & 4.66 & 4.64 \\
\hline
\end{tabular}

*work functions of SWNTs from Ref $\overline{\overline{17}}]$
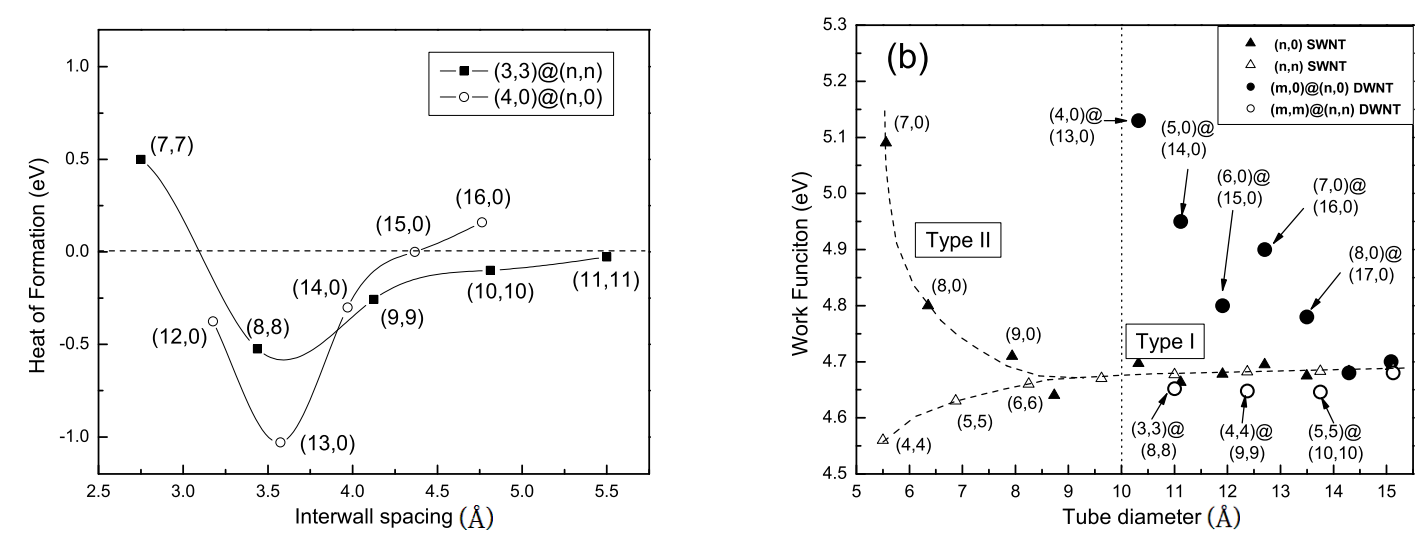

FIG. 1: (a)Heat of formation per unit cell for the DWNTs. solid square for $(3,3) @(n, n)$ DWNTs and empty circle for $(4,0) @(n, 0)$ DWNTS. (b)Work functions for DWNTs (dots) and SWNTs(triangles) of different diameters 

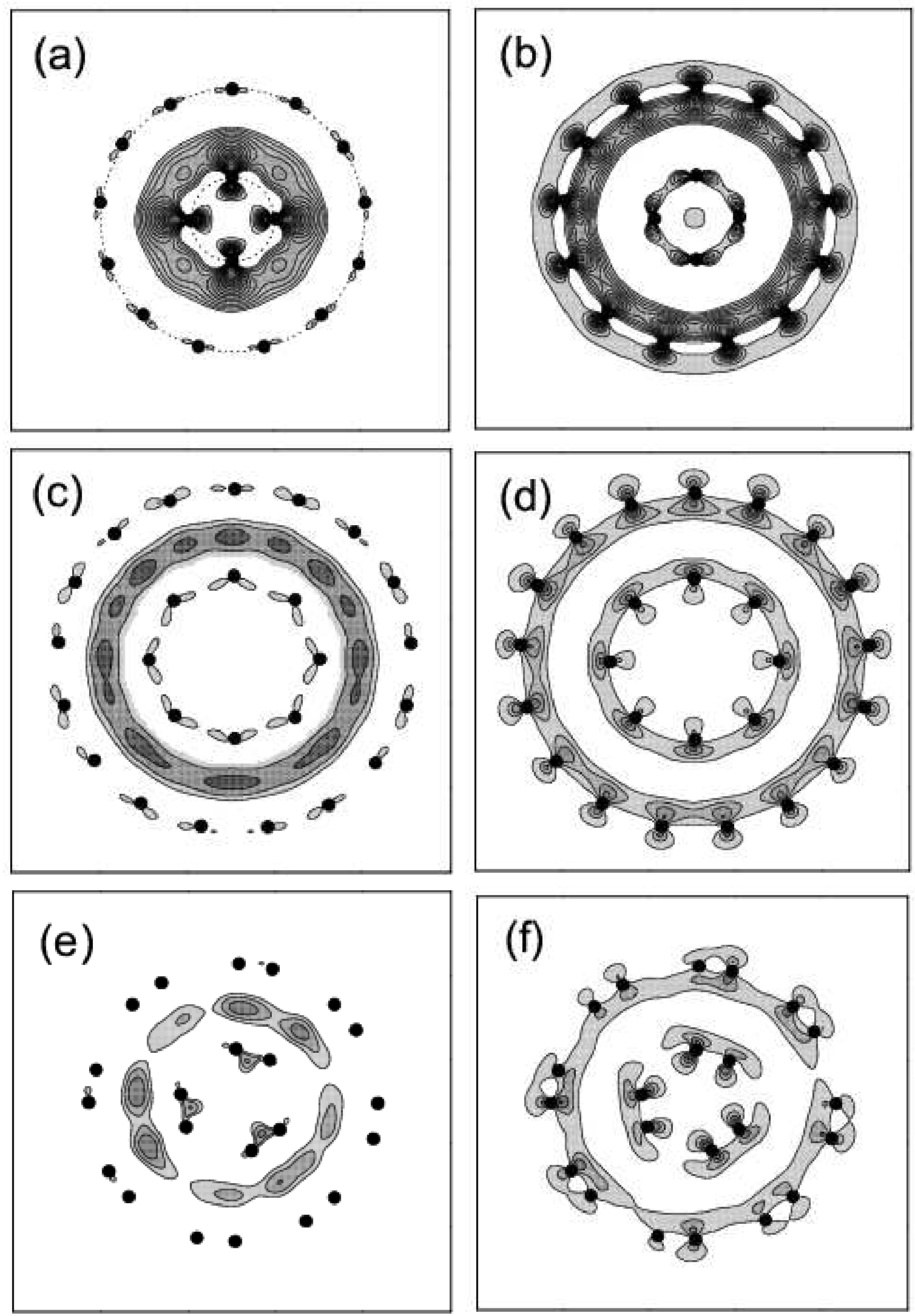

FIG. 2: Contour plots of charge accumulation (left column) and depletion region (right column) for $(4,0) @(13,0),(8,0) @(17,0)$ and $(3,3) @(8,8)$ DWNTs, respectivly. The contour plots are shown 10 in a plane normal to the tube axis, with black dots indicating positions of carbon atoms. The 

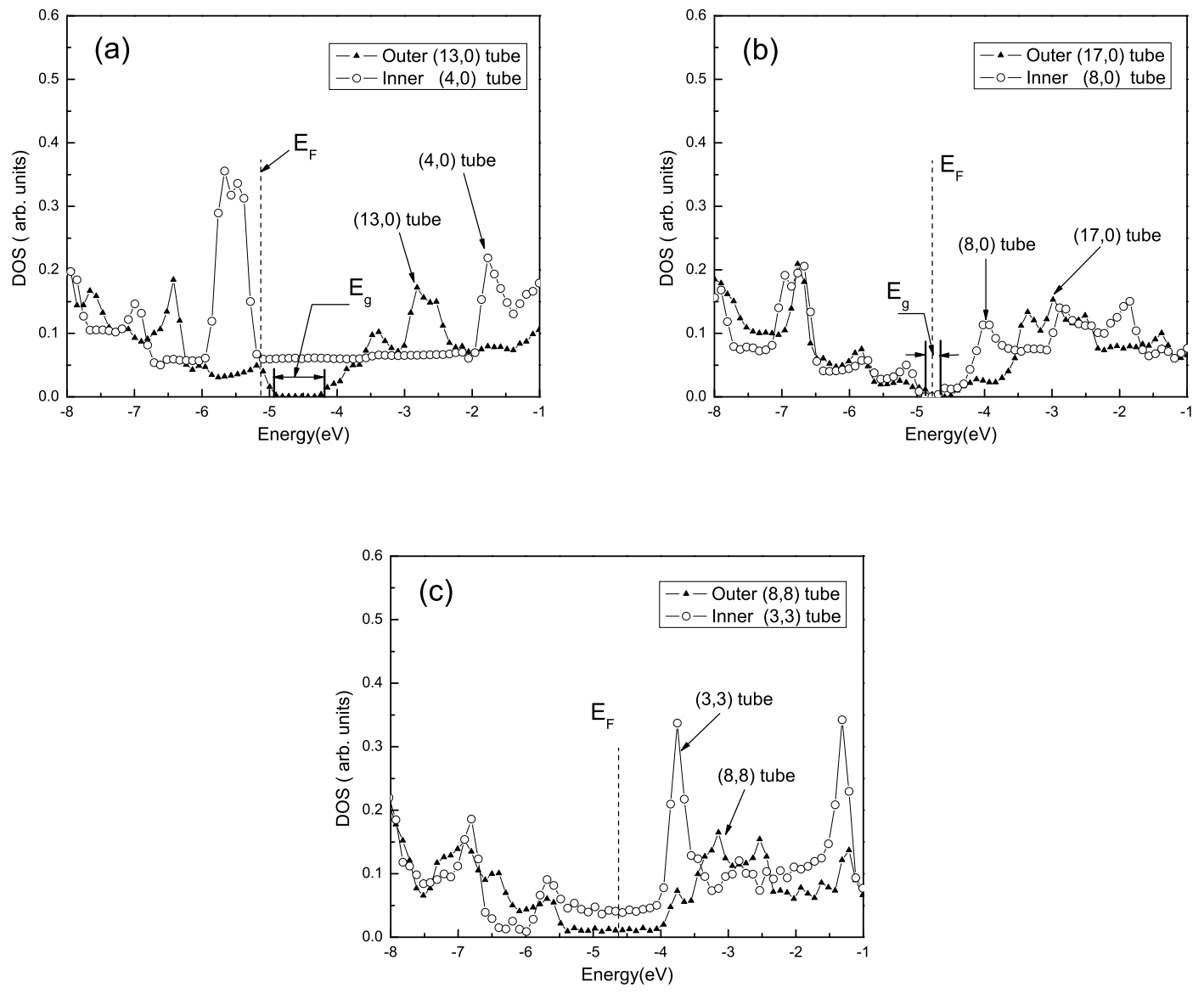

FIG. 3: Site-projected DOS for $(4,0) @(13,0),(8,0) @(17,0)$ and $(3,3) @(8,8)$ DWNTs respectively. Empty circles for the outer tube and filled triangles for the inner tube. 